\title{
Protezione ambientale: abbandono dell'antropocentrismo giuridico e evoluzione del Diritto
}

\author{
Environmental protection: abandonment \\ of legal anthropocentrism and evolution of Law
}

Livio Perra ${ }^{1}$

Riassunto: Il diritto non è statico, ma si evolve continuamente. In materia ambientale questa evoluzione sta andando nella direzione dell' abbandono dell'antropocentrismo giuridico. L'autore osserva il successo in vari luoghi del Pianeta del riconoscimento della natura o delle sue entità come soggetti o persone, con veri e propri diritti. A tal fine, egli dapprima analizza numerose di queste esperienze. Questo nuovo modo di pensare alla natura e alle sue entità avviene a livello costituzionale, legislativo, giurisprudenziale. L'autore alla luce dell'esame del fenomeno, riflette sul fatto che ora sia il momento adatto per superare la visione antropocentrica del diritto. Egli poggia tale affermazione su due fattori. Il primo è costituito

1 Professore a contratto di Legislazione dei Beni Culturali, Università degli Studi di Sassari, Italia (A.A. 2019/2020). Pesquisador Associado, Núcleo V, Democracia, Direito Internacional e Direitos Humanos do Centro Internacional de Direitos Humanos de São Paulo, vinculado à Cadeira San Tiago Dantas, da Academia Paulista de Direito, CIDHSP/APD, Brasil (BIÊNIO 2019-2021). ORCID: https://orcid.org/0000-0002-1045-1206 
dal mutamento del modo in cui l'essere umano concepisce se stesso e l'autore parla a tal proposito di Homo Ecologicus. Il secondo fattore è strettamente legato alle società odierne. L'apertura verso la pari dignità di ogni popolo che compone ogni Paese ha portato all'emergere nei dibattiti politici e giuridici della sensibilità ambientale della tradizione indigena. Inoltre, questo cambio di prospettiva non sarebbe stato possibile senza una sensibilità ambientale diffusa all'interno della società e se la previsione dei diritti di entità naturali non umane non fosse stata ritenuta una risposta efficace alla problematica ambientale.

Parole chiave: Diritti della natura. Soggetto di diritti. Persona giuridica. Homo Ecologicus. Società.

Abstract: Law is not static, but continuously evolves. In environmental matters, this evolution is going in the direction of abandoning legal anthropocentrism. The author observes the success in various places on the Planet of recognising nature or its entities as subjects or people, with real rights. For this purpose, he first analyses several of these experiences. This new way of thinking about nature and its entities takes place at a constitutional, legislative and jurisprudential level. In the light of examining the phenomenon, the author reflects on the fact that now is the right time to overcome the anthropocentric vision of law. He rests this claim on two factors. The first is constituted by the change in the way in which the human being conceives himself and the author speaks in this regard of Homo Ecologicus. The second factor is closely related to today's societies. The openness towards the equal dignity of every people that makes up each Country has led to the emergence of the environmental sensitivity of the indigenous tradition in political and legal debates. Furthermore, this change of perspective 
would not have been possible without a widespread environmental sensitivity within society and if the provision of the rights of non-human natural entities had not been considered an effective response to the environmental issue.

Keywords: Rights of nature. Subject of rights. Legal person. Homo Ecologicus. Society.

\section{Introduzione}

Nel mondo giuridico ogni fenomeno riceve una propria collocazione, interpretazione e sistemazione in base alla visione del mondo che caratterizza una determinata società. Il diritto, come afferma Jean Cruet: "non domina la società, ma l'esprime ${ }^{\prime \prime 2}$. Ora, occorre prendere spunto dalla poetica immagine descritta da Philippe Descola ${ }^{3}$, il quale osservando il Museo di Storia Naturale de la ciudad de La Plata, sottolinea che l'intera esposizione è organizzata seguendo una data sistemazione che esprime l'idea di ordine del mondo. Trasportando questa rappresentazione nel mondo del diritto, occorre vedere su cosa essa poggi. Le classificazioni e le categorie non sempre possono comprendere tutti i nuovi fenomeni che sorgono nel mondo giuridico nel loro alveo o faticano nell'intento. Quando ci si accorge che non vi sono categorie a cui ricondurre i fenomeni o in cui classificarli, sorgono nuove categorie.

Riprendendo la metaforica descrizione delle esposizioni nel museo, giova tener presente che coloro i quali curano le esposizioni nei musei, spesso aggiornano la disposizione degli oggetti e rivisitano l'ordine dei settori e delle zone

2 CRUET, 1908, p. 336: "Le droit ne domine pas la société, il l'exprime". Si veda a tal proposito: VIOLA, 2018, p. 10.

3 Si veda a tal proposito: DESCOLA, 2011, pp. 76-77. 
espositive. Allo stesso modo, il diritto si trova dinnanzi alla costante sfida di aggiornarsi ed evolversi, in un quadro di costante dinamismo, per rispondere prontamente alle esigenze sorte in seno alla società.

L'evoluzione del diritto si rende necessaria se si intende il diritto come manifestazione del sentire sociale e non solo come forza ordinante il vivere sociale. I legislatori, i giudici e gli avvocati avvertono spesso questa esigenza di adeguare il diritto alle esigenze manifestate dalla società ed in tal modo sorgono nuove norme a regolare alcune materie e nascono interpretazioni più adatte delle disposizioni giuridiche già presenti nello scenario giuridico. Essi sanno che si deve fuggire dalla cristallizzazione e staticità portate all'estremo, nulla è più pericoloso del modo di dire "abbiamo sempre fatto così". In tal modo, come occorre cambiare marcia quando una automobile presenta i suoi limiti nell'affrontare una salita impegnativa, il diritto nell'allontanamento dalle prospettive antropocentriche trova la spinta per rinnovarsi, perfezionarsi, evolversi e migliorarsi.

In materia ambientale si assiste oggi ad una sfida importante: di giorno in giorno si moltiplicano gli ordinamenti giuridici dove viene avvertita la necessità dell'abbandono dell' antropocentrismo giuridico per rispondere alla crescente esigenza manifestata dalla società di una tutela ambientale più efficace.

\section{Da oggetto a soggetto}

In una visione del diritto dovedominal'antropocentrismo tutto è previsto in funzione dell'essere umano. La natura e le sue entità sono considerate oggetti. In vari Paesi dalla società arrivano costanti istanze che vogliono liberare la natura e le sue componenti da questa asfittica morsa che le relega nel mondo delle res. In vari Paesi questa spinta verso 
la personificazione giunge dalle istanze portate avanti dai popoli che abitano i territori e reclamano una maggiore tutela ambientale. In particolare, è chiesto il riconoscimento giuridico di soggetto o persona alla natura e alle sue componenti, nell'ottica di una importanza già presente nella cultura, nella tradizione e nella visione del mondo dei popoli indigeni che compongono lo Stato, oppure la società chiede il riconoscimento di una sensibilità ambientale maturata al proprio interno.

Queste istanze sono accolte in numerosi Paesi, dove la natura diviene sujeto de derechos ed i fiumi o altre entità naturali sono legal persons. Il diritto, in questo modo, si trova dinnanzi ad una importante sfida: un cambio di prospettiva che tenga conto dell'esistenza di nuovi soggetti non umani del mondo naturale.

Gli esempi osservabili del fenomeno sono numerosi ed assumono varie forme. La natura o le sue entità sono riconosciute come soggetti o persone nelle Costituzioni, nelle leggi, nelle ordinanze municipali, nelle sentenze e nei progetti di legge.

In Ecuador, la Costituzione del 2008 afferma nell'articolo 10 , al secondo comma, che: "la natura sarà soggetto di quei diritti che le riconosce la Costituzione" ${ }^{\prime 4}$. Negli articoli 71 e 72 della medesima Costituzione sono previsti i diritti della natura, distinti tra i diritti che concernono l'esistenza e quelli tesi alla restauración.

In Bolivia, la Ley de derechos de la Madre Tierra (n. 71 del 2010), nell' articolo 5 definisce la natura come "soggetto collettivo di interesse pubblico" ${ }^{5}$. Nell'articolo 7 della stessa legge sono affermati i diritti alla vita ${ }^{6}$, alla diversità della

4 "La naturaleza será sujeto de aquellos derechos que le reconozca la Constitución".

5 "Sujeto colectivo de interés público".

6 "A la vida". 
vita $^{7}$, all' acqua ${ }^{8}$, all'aria pulita ${ }^{9}$, all'equilibrio ${ }^{10}$, al ripristi$\mathrm{no}^{11}$, a vivere senza contaminazioni ${ }^{12}$. Oltre a questo elenco, nell'articolo 5 della stessa legge è affermato che potranno essere previsti altri ed ulteriori diritti.

In Nuova Zelanda, il Whanganui River [Te Awa Tupua] è riconosciuto come essere vivente, tramite un accordo stipulato il 5 agosto 2014 tra i rappresentanti del popolo Whanganui River Iwi ed il governo neozelandese ${ }^{13}$. Con ulteriori accordi ed atti il medesimo fiume viene riconosciuto come legal person ${ }^{14}$.

Sempre in Nuova Zelanda, nel 2014 il parco nazionale Te Urewera diviene una entità legale ${ }^{15}$ con i diritti, i poteri, i doveri e le responsabilità di una persona giuridica ${ }^{16}$.

In Australia, il fiume Yarra con i territori limitrofi viene definito come entità naturale vivente e integrata ${ }^{17}$.

In Colombia, nella sentenza T-622 del 2016 della Corte Costituzionale il fiume Atrato diviene sujeto de derechos, compresi il suo bacino ed affluenti. Saranno lo Stato e le comunità etniche, come previsto nella stessa sentenza, ad esercitare la tutela e la rappresentanza legale dei diritti del fiume e ciò al fine dell'attuazione concreta della protezione

\footnotetext{
7 "A la diversidad de la vida".

8 "Al agua".

9 "Al aire limpio".

10 "Al equilibro".

11 "A la restauración".

12 "A vivir libre de contaminación".

13 Si veda a tal proposito: SALMOND, 2014, pp. 285-286.

14 Si veda a tal proposito: Te Awa Tupua (Whanganui River Claims Settlement) Act 2017.

15 "Legal entity".

16 Si veda a tal proposito: Te Urewera Act 2014.

17 "Living and integrated natural entity". Si veda a tal proposito: Yarra River Protection (Wilip-gin Birrarung murron) Act 2017.
} 
del fiume e della restaurazione di esso in conseguenza dei danni verificatisi ${ }^{18}$.

Nello Stato di Uttarakhand, in India, nel 2017 la High Court of Uttarakhand at Nainital afferma che i fiumi Ganga e Yamuna sono legal persons/living persons ${ }^{19}$.

La High Court Division of the Supreme Court of Bangladesh riconosce nel 2019 il fiume Turag e tutti i fiumi del Paese entità vive con personalità giuridica ${ }^{20}$.

Nella Constitución Política de la Ciudad de México, nel 2017, la natura con i suoi ecosistemi e specie diviene, nell'articolo 13, "ente collettivo soggetto di diritti" ${ }^{21}$.

Nella Constitución Política del Estado Libre y Soberano de Guerrero nell'articolo 2 è affermato che: "lo Stato dovrà garantire e proteggere i diritti della natura nella relativa legislazione" 22 .

In Brasile, a livello municipale sono osservabili due esempi nello Stato di Pernambuco. Il 21 dicembre 2017 la Câmara Municipal do Bonito modifica l'articolo 236 della Lei Orgânica numero $01 / 2017$ riconoscendo alla natura il diritto di esistere, prosperare e evolversi ${ }^{23}$. Il 2 maggio 2018 la $C \hat{a}-$

18 Si veda a tal proposito: Sentenza T-622/16 della Corte Constitucional de Colombia, 159, punto 10.2.

19 Si veda a tal proposito: Mohd. Salim v. State of Uttarakhand $\mathcal{E}$ others, Writ Petition (PIL) No. 126 of 2014 (High Court of Uttarakhand at Nainital, March 20, 2017), p. 11; PERRA, 2020, p. 347.

20 Si veda a tal proposito: Human Rights and Peace for Bangladesh Vs Bangladesh and others, Writ Petition 13989/2016 (High Court Division of the Supreme Court of Bangladesh, July 1, 2019).

21 "Ente colectivo sujeto de derechos".

22 "El Estado deberá garantizar y proteger los derechos de la naturaleza en la legislación respectiva". Si veda a tal proposito: PERRA, 2018, p. 15.

23 Si veda a tal proposito l'articolo 236 della Lei Orgânica numero 01/2017, il quale afferma che: "o Município reconhece o direito da natureza de existir, prosperar e evoluir, e deverá atuar no sentido de assegurar a todos os membros da comunidade natural, humanos e não humanos, do Município de Bonito, o direito 
mara Municipal do Paudalho modifica l'articolo 181 della Lei Orgânica numero 03/2018, riconoscendo alla natura il diritto di esistere, prosperare ed evolversi ${ }^{24}$.

Inoltre, va sottolineato il fatto che il Superior Tribunal de Justiça del Brasile ${ }^{25}$ ha rilevato la necessità di una riflessione nel campo interno che porti ad una matura discussione sul riconoscimento degli animali non umani come soggetti con propri diritti.

In Argentina, nel 2014 la Cámara Federal de Casación Penal, Capital Federal, Ciudad Autónoma de Buenos Aires, nella causa conosciuta come "orangotango Sandra", riconosce l'animale come soggetto non umano titolare di diritti ed in tal modo viene ammesso che vi siano soggetti non umani che abbiano propri diritti ${ }^{26}$.

Negli Stati Uniti d'America, lo Stato del Colorado nel 2014 approva un emendamento costituzionale con cui aggiungere nell'articolo 2 la sezione 32. In questa parte viene stabilito che le persone nel quadro del diritto locale all' autogoverno possano, nelle contee e nelle municipalità, emanare leggi al fine di stabilire, proteggere e garantire i diritti degli

ao meio ambiente ecologicamente saudável e equilibrado e à manutenção dos processos ecossistêmicos necessários à qualidade de vida, cabendo ao Poder Público e à coletividade, defendê-lo e preservá-lo, para as gerações presentes e futuras dos membros da comunidade da terra".

24 Si veda a tal proposito l'articolo 181 della Lei Orgânica numero 03/2018, il quale afferma che: "o município reconhece o direito da Natureza existir, prosperar e evoluir e deverá atuar no sentido de assegurar a todos os membros da comunidade natural, humanos e não humanos, do município do Paudalho, o direito ao meio ambiente ecologicamente saudável e equilibrado e a manutenção dos processos ecossistêmicos necessários à qualidade da vida, cabendo ao município e à coletividade, defendê-lo e preservá-lo para as gerações presentes e futuras dos membros da comunidade da Terra".

25 Si veda a tal proposito: Superior Tribunal de Justiça do Brasil, Recurso Especial 1.797.175 - SP, pp. 9-19.

26 Si veda a tal proposito: Cámara Federal de Casación Penal, Capital Federal, Ciudad Autónoma de Buenos Aires, Causa numero CCC 68831/2014/CFC1. 
individui, delle comunità e della natura. L'iter di questo emendamento non ha avuto seguito.

Nel 2010 nell'articolo 1 del The Pittsburgh Code viene inserito il Chapter 618 [Marcellus Shale Natural Gas Drilling]. In particolare, i residenti, le comunità naturali e gli ecosistemi di Pittsburgh possiedono un diritto fondamentale e inalienabile di accesso sostenibile, uso, consumo e conservazione dell' acqua $^{27}$. Vi sono, inoltre, previsti i diritti inalienabili e fondamentali relativi all'esistenza ed al prosperare nella città di Pittsburgh in capo alle comunità naturali e agli ecosistemi. I cittadini di Pittsburgh agiranno in nome delle entità naturali per far valere questi diritti ${ }^{28}$.

In Pennsylvania, nel Tamaqua Borough, Schuylkill County, con l'Ordinance numero 612 del 2006, nella Section 7.6, le comunità naturali e gli ecosistemi sono riconosciuti come persone ai fini dell'applicazione dei diritti civili ${ }^{29}$.

In Colorado, a Lafayette viene affermato, nella Section 1(a) dell'Ordinance numero 02, Series 2017, il diritto dei residenti e degli ecosistemi ad un clima sano ${ }^{30}$.

27 Si veda a tal proposito il The Pittsburgh Code, Chapter 618.3 a): "Right to Water. All residents, natural communities and ecosystems in Pittsburgh possess a fundamental and inalienable right to sustainably access, use, consume, and preserve water drawn from natural water cycles that provide water necessary to sustain life within the City".

28 Si veda a tal proposito il The Pittsburgh Code, Chapter 618.3 b): "Rights of Natural Communities. Natural communities and ecosystems, including, but not limited to, wetlands, streams, rivers, aquifers, and other water systems, possess inalienable and fundamental rights to exist and flourish within the City of Pittsburgh. Residents of the City shall possess legal standing to enforce those rights on behalf of those natural communities and ecosystems".

29 Si veda a tal proposito Tamaqua Borough, Schuylkill County, Pennsylvania, Ordinance n. 612 of 2006, Section 7.6: "Borough residents, natural communities, and ecosystems shall be considered "persons" for the purposes of the enforcement of the civil rights of those residents, natural communities, and ecosystems".

30 Si veda a tal proposito: Lafayette Climate Bill of Rights, Section 1(a): "Right to a Healthy Climate. All residents and ecosystems of the City of Lafayette possess 
In Ohio, la città di Toledo promulga nel 2019 la Carta dei diritti del lago Erie [Lake Erie Bill of Rights], nella quale vengono riconosciuti i diritti dell'ecosistema del lago Erie.

In Uganda, la prima parte dell'articolo 4 del The National Environment Act 2019 stabilisce che "la natura ha il diritto di esistere, persistere, mantenere e rigenerare i suoi cicli vitali, struttura, funzioni e i suoi processi evolutivi" ${ }^{31}$. Nel medesimo articolo è, inoltre, previsto che le persone possono agire dinnanzi alla corte competente per le violazioni dei diritti della natura ${ }^{32}$.

Si osservano, infine, due progetti di legge. Il primo presentato in Argentina dai senatori Fernando Ezequiel "Pino" Solanas e Rubén Héctor Giustiniani: Proyecto de Ley sobre Derechos de la Naturaleza (Nro. de Expediente 2506/15). Questo progetto menziona tra i fondamenti anche l'esempio offerto dalle esperienze di Ecuador e Bolivia, dal quale i proponenti affermano di aver tratto ispirazione per il loro progetto di legge. In tale testo sono previsti espressamente i diritti della natura. Il secondo progetto è presentato in Colombia dal Representante a la Cámara Juan Carlos Lozada Vargas. In tale progetto sono riportate le esperienze di alcuni Stati che hanno stabilito in varie forme che la natura o le sue entità sono soggetti o persone con veri e propri diritti. Con tale progetto è richiesta la modifica dell'articolo 79 della

a right to a healthy climate and life sustaining resources, which shall include the right to be free from all activities within the City of Lafayette that interfere with that right, including the extraction of coal, oil, or gas, disposal of drilling waste, contaminated drinking water, lethal carcinogens, toxic gases and other byproducts of industrial activity which threaten human physical and neurological systems".

31 Si veda a tal proposito il The National Environment Act 2019, articolo 4 (1): "Nature has the right to exist, persist, maintain and regenerate its vital cycles, structure, functions and its processes in evolution".

32 Si veda a tal proposito il The National Environment Act 2019, articolo 4 (2): "A person has a right to bring an action before a competent court for any infringement of rights of nature under this Act". 
Constitución Política de Colombia per "includere i diritti della natura" 33 e riconoscere la natura come "una entità vivente soggetto di diritti" ${ }^{34}$.

\section{Homo Ecologicus}

L'essere umano, concepito antropocentricamente come il centro del suo universo, si trova ad affrontare una seria riflessione riguardo al proprio posto nel mondo.

Nelle nuove concezioni che predicano i diritti della natura e delle sue entità, l'uomo ha compreso di non essere l'unico soggetto o persona. Egli non può dominare la natura a suo piacimento. La natura e le sue componenti acquistano per il diritto una nuova dignità, quella che deriva dal riconoscimento come soggetti o persone. Gli individui si trovano in questo modo inseriti in un contesto più ampio, contribuiscono con tutte le entità naturali a formare un tutto vivo ed il benessere del tutto dipende da quello di ciascuna parte.

La previsione di veri e propri diritti della natura o delle sue componenti comporta una modifica non solo di prospettiva o del modo di vedere il mondo. Si tratta di un cambiamento negli stili di vita e nello stesso modo di concepire l'uomo. L'essere umano deve rispettare i nuovi diritti emersi. La natura o le sue entità spesso sono sostituite dagli individui con strumenti definiti come rappresentanza o tutela per innescare i meccanismi previsti nei nuovi sistemi di protezione ambientale che portino ad una efficace ed effettiva tutela.

L'uomo in tal contesto assume comportamenti più responsabili ed ecosostenibili. Non vi è una spinta verso un ecologismo estremo, ma l'intento dei nuovi sistemi è quello

33 "Los derechos de la naturaleza".

34 "Una entidad viviente sujeto de derechos". 
limitare l'impatto dell'essere umano nel mondo circostante. Il rispetto dei diritti della natura e delle sue entità comporta l'abbandono di pratiche umane che sfruttano le risorse naturali al di là di ogni ragionevole limite. Sono scoraggiati gli sprechi ed i comportamenti che vedono in ciò che offre la natura uno strumento con cui raggiungere un guadagno economico che prescinda dalla sua salvaguardia.

Si affaccia nel mondo giuridico l'idea di Homo Ecologicus, l'uomo modello è quello virtuoso, che conscio del valore rivestito dalla natura e dalle sue entità, rispettando i diritti ecologici ${ }^{35}$, impronta i propri comportamenti a stili di vita atti a tutelare il mondo che lo circonda e instaura una relazione armoniosa con la natura e le sue entità.

I nuovi sistemi di protezione ambientale non sono soltanto fondati su un insieme di diritti attribuiti ad entità naturali non umane e sugli strumenti per farli rispettare, non si tratta solo di sanzionare o reprimere le violazioni di tali diritti. Accanto alla previsione di questi sistemi, i Paesi si preoccupano di sensibilizzare ed informare i cittadini sulle buone pratiche.

\section{I tempi sono pronti}

Nell'epoca attuale le preoccupazioni sulla salute dell'ambiente crescono ogni giorno. I più grandi disastri che minano il benessere della natura destano scalpore, le notizie affollano lo scenario televisivo globale e la carta stampata ${ }^{36}$ e lanciano importanti campanelli d'allarme. La rovina della natura è una presenza costante, anche quando non appare in queste espressioni drammatiche. La sopravvivenza del

35 Alberto Acosta (2011, pp. 354-355) distingue i diritti della natura, intesi come diritti ecologici, da quelli ambientali che derivano dai diritti umani.

36 Si veda a tal proposito PERRA, 2020, p. 339. 
Pianeta è minacciata anche attraverso forme silenti e non sempre eclatanti. Dinnanzi a tali preoccupazioni, ogni Paese è alla continua ricerca di soluzioni per difendere la natura da svariati attacchi che ne minano la stabilità e gli equilibri. Alcuni Stati escogitano la soluzione del riconoscimento giuridico della natura o delle sue entità come soggetti o persone titolari di veri e propri diritti. Operare una simile scelta, tra il ventaglio di soluzioni possibili, si presta essenzialmente a due rilevanti considerazioni:

- Si tratta di una scelta che porta ad una efficace tutela;

- È il frutto di un dibattito politico e giuridico che vede in tale soluzione la scelta più adatta.

Sull'efficacia di questa scelta è osservabile che i sistemi di protezione della natura che riconoscono i diritti della stessa o delle sue entità, predispongono anche i necessari meccanismi e strumenti perché la tutela sia concreta ed i diritti non restino soltanto affermati su carta. L'ampia gamma di azioni, e, più in generale, i meccanismi volti ad assicurare l'effettivo rispetto dei diritti della natura e delle sue componenti all'esistenza e alla restaurazione nel caso in cui si verifichino danni, è completata dalla previsione di strumenti assimilati alla rappresentanza o alla tutela ${ }^{37}$ (in base al sistema giuridico di riferimento), con i quali l'essere umano sopperisce all' incapacità della natura o delle sue entità di curare i propri interessi o di adire i competenti tribunali.

Il maggior vantaggio di tali sistemi è la versatilità nella difesa della natura e delle sue entità. Non occorrono norme che prescrivano determinati comportamenti o ne vietino altri, ma le violazioni dei diritti affermati trovano

37 Si veda a tal proposito: ÁVILA SANTAMARÍA, 2010, pp. 200-202. 
una pronta risposta dell'ordinamento. Sono numerose le sentenze che offrono segnali in questo senso. Dalle aule dei tribunali negli anni a venire, in un arco di tempo osservabile più ampio rispetto a quello oggi a disposizione, costituito solo da poco più di un decennio, emergeranno numerosi dati ed un fecondo campo d'analisi dove potrà essere verificata la portata dell'efficacia dei sistemi di protezione ambientale che prevedono i diritti della natura e delle sue entità.

La seconda considerazione che può essere effettuata è legata alla genesi dei sistemi di protezione ambientale incentrati sulla previsione dei diritti della natura e delle sue entità.

Questi sistemi sono nati a seguito di importanti dibattiti politici e giuridici. All' esito di tali dibattiti sono state accolte le istanze portate avanti dalla società che chiedevano il riconoscimento della natura o delle sue entità come soggetti o persone e la conseguente attribuzione di veri e propri diritti. L'interrogativo che sorge spontaneo è relativo al momento in cui ciò è accaduto, cioè " perché ora?" ed occorre a tal fine analizzare il clima in cui queste nuove concezioni hanno visto il proprio emergere.

In alcuni casi l'affermazione di questa nuova sensibilità ambientale è dovuta all'intensa attività dei movimenti e dei partiti indigeni, alle riflessioni portate in campo accademico dagli studiosi indigeni. Nello scenario in cui le democrazie odierne superano i preconcetti di epoca coloniale, che suddividevano le culture su una scala di importanza e consideravano quelle indigene come subculture, esse danno pari dignità a tutti i popoli ed etnie che abitano l'intero territorio del Paese. In questo modo, ogni popolo può portare avanti il bagaglio di valori della sua tradizione e chiederne il riconoscimento. Dalla pari dignità data alla voce di ogni singolo individuo o gruppo deriva un arricchimento per il dibattito politico e giuridico. Occorre considerare che i valori culturali 
del rispetto della natura trovano terreno fertile nella società, basti pensare al fatto che questi valori e le loro possibili applicazioni formano oggetto di numerosi studi anche tra gli studiosi non appartenenti a determinate etnie o gruppi indigeni. Infatti, in seno alle medesime società odiernamente si sta affermando sempre più una certa sensibilità ambientale, una attenzione particolare verso l'equilibrio della natura e dei suoi cicli vitali. A tal fine, si cercano nuovi sistemi e strumenti per correggere gli squilibri provocati dall'azione e dall'impatto dell'essere umano sull' ambiente che lo circonda. Allo stesso tempo, rispetto al passato, è cresciuta considerevolmente la consapevolezza che i danni inferti all' ambiente possono ripercuotersi sulla salute ed il benessere globale e per questo motivo si moltiplicano gli avvertimenti ed i consigli utili per incoraggiare le buone pratiche per scongiurare gravi e seri danni ambientali presenti e futuri.

\section{Conclusioni}

In alcuni Paesi emergono nuovi modelli di protezione ambientale, dove la natura o le sue entità divengono soggetti o persone con veri e propri diritti. Il riconoscimento delle entità naturali non umane come soggetti o persone costituisce un inedito nel mondo giuridico. Per comprendere come ciò sia avvenuto, nel presente lavoro sono stati osservati i fenomeni emersi in diversi Paesi. Sono vari fattori alla base dell'abbandono dell' antropocentrismo nello scenario giuridico dove è stata accolta la personificazione giuridica della natura o delle sue entità e la conseguente previsione di diritti con gli appositi strumenti per garantirli.

In primo luogo si è volto lo sguardo alla trasposizione dei valori delle culture indigene e della sensibilità ambientale emersa nella nostra epoca e sul fatto che questa 
nuova ventata di idee nel diritto ha potuto accedervi grazie all' impostazione democratica dei Paesi. In un quadro dove è predicata la pari dignità dei popoli che compongono i territori nazionali, ogni individuo o comunità può contribuire alle riflessioni che confluiscono nei dibattitti politici e giuridici.

In secondo luogo, si è voluto evidenziare che il clima in cui il diritto ha rispolverato tutto il proprio dinamismo per andare incontro alle istanze di tutela ambientale, portate avanti dalla società, dai movimenti indigeni e dai partiti, è caratterizzato da un nuovo concetto di individuo, inteso come Homo Ecologicus. Si constata che la società oggi ha al suo interno la sensibilità non solo per richiedere l'abbandono dell' antropocentrismo giuridico, ma addirittura per richiedere essa stessa il riconoscimento della natura o delle sue entità come soggetti o persone e la previsione di veri e propri diritti ecologici.

Optare per un modello di protezione ambientale che preveda i diritti della natura o delle sue entità diviene una possibile via verso una tutela efficace delle entità naturali, in quanto ogni fenomeno che si ponga in contrasto con $i$ medesimi diritti trova una pronta risposta da parte degli ordinamenti giuridici. Inoltre, la scelta sta riscuotendo un discreto successo e, come dimostra la sua diffusione in luoghi diversi del Pianeta, anche distanti culturalmente e geograficamente, parrebbe che essa sia ritenuta una strada percorribile verso una adeguata difesa ambientale.

Il mondo giuridico per rispondere alla problematica ambientale sta mettendo in discussione la concezione secondo cui tutto è pensato in funzione dell'essere umano. In una realtà dove nella società emergono nuove sensibilità ambientali, dove alcuni popoli che compongono i Paesi chiedono a gran voce un adeguamento alle cosmovisioni portate avanti, il diritto non può rimanere fermo. Esso deve 
evolversi, recepire i nuovi valori e dare ordine all'intero sistema alla luce delle novità introdotte. Le categorie tradizionali devono essere rivisitate, adattate, ne sorgono di nuove e ciò costituisce l'evoluzione nel mondo giuridico. La cristallizzazione del diritto è un limite all'adeguarsi al sentire sociale che deve essere superato. Il mondo giuridico deve essere pronto a rispondere alle nuove esigenze che emergono ed assecondare il cambiamento.

Le democrazie odierne divengono in tal modo espressione dei valori ritenuti importanti dalla società e così facendo il diritto assolve alla propria funzione di regolare il vivere sociale secondo un sistema più prossimo alla visione del mondo dei popoli che compongono i Paesi.

Si è dinnanzi alla maturata consapevolezza che i danni ambientali, provocati dallo sconsiderato sfruttamento delle risorse naturali oltre ogni limite ragionevole e tendente al solo profitto economico, possono condurre alla rovina del Pianeta e minacciare la stessa esistenza umana. La società, pertanto, chiede risposte serie e puntuali per difendere l'ambiente.

Tra la vasta gamma di soluzioni possibili alcuni Paesi hanno accolto nelle proprie riflessioni politiche e giuridiche i valori culturali dei popoli indigeni che abitano i loro territori e, più in generale, la nuova sensibilità ambientale sviluppatasi negli anni all'interno della società. Riscoperta o trovata l'armonia con la natura, alcuni ordinamenti giuridici costruiscono sistemi di protezione ambientale dove la natura o le sue entità hanno propri diritti, traendo ispirazione dalle culture che vedono nella natura e nelle sue componenti una sacralità talmente alta da vedere le stesse assurgere a veri e propri soggetti o persone. 


\section{Bibliografia}

ACOSTA, Alberto. Los Derechos de la Naturaleza. Una lectura sobre el derecho a la existencia. In: ACOSTA, Alberto; MARTÍNEZ, Esperanza (orgs.). La naturaleza con derechos. De la filosofía a la política. Quito: Abya-Yala. pp. 317-367, 2011. ÁVILASANTAMARÍA, Ramiro. El derecho de la naturaleza: fundamentos. In ACOSTA, Alberto; MARTÍNEZ, Esperanza (orgs.). La naturaleza con derechos. De la filosofía a la política. Quito: Abya-Yala. pp. 173-238, 2011.

BAVIKATTE, Kabir Sanjay; BENNETT, Tom. Community stewardship: the foundation of biocultural rights. In: Journal of Human Rights and the Environment, v. 6, n. 1, pp. 7-29, 2015. Disponível em: <http:/ / www.elgaronline.com/view/journals/jhre/6-1/jhre.2015.01.01.xml>. Acesso em: 5 abril 2020.

BERROS, María Valeria. Ética animal en diálogo con recientes reformas en la legislación de países latinoamericanos. In: Revista de Bioética y Derecho, n. 33, pp. 82-93, 2015. Disponível em: <http:/ / revistes.ub.edu/index.php/RBD/article/ view/11566>. Acesso em: 5 abril 2020.

CRUET, Jean. La vie du droit et l'impuissance des lois. Paris: Ernest Flammarion, 1908.

CRUZ RODRÍGUEZ, Edwin. Derechos de la naturaleza, descolonización e interculturalidad. Acerca del caso ecuatoriano. In: Verba Iuris, n. 31, pp. 15-29, 2014. Disponível em: <http:/ / revistas.unilibre.edu.co/index.php/verbaiuris/ article/view/53>. Acesso em: 5 abril 2020.

DESCOLA, Philippe. Más allá de la naturaleza y de la cultura. In: MONTENEGRO MARTÍNEZ, Leonardo (org.). Cultura y Naturaleza. Aproximaciones a propósito del bicentenario 
de la independencia de Colombia. Bogotá: Jardín Botánico de Bogotá, José Celestino Mutis. pp. 76-98, 2011.

GUDYNAS, Eduardo. Desarrollo, derechos de la naturaleza y Buen vivir despues de Montecristi. In: WEBER, Gabriela (org.). Debates sobre cooperación y modelos de desarrollo. Perspectivas desde la sociedad civil en el Ecuador. Quito: Centro de Investigaciones CIUDAD y Observatorio de la Cooperación al Desarrollo. pp. 83-102, 2011a.

GUDYNAS, Eduardo. Los derechos de la Naturaleza en serio. Respuestas y aportes desde la ecología política. In: ACOSTA, Alberto; MARTÍNEZ, Esperanza (orgs.). La naturaleza con derechos. De la filosofía a la política. Quito: Abya-Yala. pp. 239-286, 2011 b.

HSIAO, Elaine C. Whanganui River Agreement - Indigenous Rights and Rights of Nature. In: Environmental Policy and Law, v. 42, n. 6, pp. 371-375, 2012.

LANDÍVAR MOSIÑO, Eric Cícero. Indigenismo y constitución en Bolivia (un enfoque desde 1990 a la fecha). In: Revista Boliviana de Derecho, n. 19, pp. 470-507, 2015. Disponível em: < http:/ / www.revista-rbd.com/numero-19/>. Acesso em: 5 abril 2020.

LOZADA VARGAS, Juan Carlos. Proyecto de Acto Legislativo "por el cual se modifica el artículo 79 de la Constitución Política de Colombia". Disponível em: <https:/ /legislapp.mininterior. gov.co/media/archivos_proyectos/PAL_080-19C.pdf $>$. Acesso em: 7 abril 2020.

MACÍAS GÓMEZ, Luis Fernando. El constitucionalismo ambiental en la nueva Constitución de Ecuador. Un reto a la tradición constitucional. In: Iuris Dictio, v. 12, n. 14, pp. 151-168, 2011. Disponível em: <http://revistas.usfq.edu. ec/index.php/iurisdictio/article/view/704>. Acesso em: 5 abril 2020. 
MARTÍNEZ, Esperanza; ACOSTA, Alberto. Los Derechos de la Naturaleza como puerta de entrada a otro mundo posible. In: Revista Direito e Práxis, v. 8, n. 4, pp. 2927-2961, 2017. Disponível em: <http:/ / www.e-publicacoes.uerj.br/ index.php/revistaceaju/article/view/31220>. Acesso em: 5 abril 2020.

MURCIA RIAÑO, Diana Milena. La naturaleza con derechos. Un recorrido por el derecho internacional de los derechos humanos, del ambiente y del desarrollo. Quito: Aurora Donoso Game, 2012.

PERRA, Livio. La natura e i suoi diritti. In: Nomos: le attualità nel diritto, n. 2, pp. 1-17, 2018. Disponível em: <http:/ / www. nomos-leattualitaneldiritto.it/nomos/livio-perra-la-natura-e-i-suoi-diritti/>. Acesso em: 5 abril 2020.

PERRA, Livio. Tradition Can Save the Future of Nature: Biocentric View of Law. In: DOBBINS, Elizabeth G.; PIGA, Maria Lucia; MANCA, Luigi (orgs.). Environment, Social Justice, and the Media in the Age of the Anthropocene. Lanham, MD: Lexington Books. pp. 339-354, 2020.

SALMOND, Anne. Tears of Rangi: Water, power, and people in New Zealand. In: Hau: Journal of Ethnographic Theory, v. 4, n. 3, pp. 285-309, 2014. Disponível em: <http://www. haujournal.org/index.php/hau/article/view/hau4.3.017>. Acesso em: 5 abril 2020.

SOLANAS, Fernando Ezequiel "Pino"; GIUSTINIANI, Rubén Héctor. Proyecto de Ley (S-2506/15). Derechos de la Naturaleza. Disponível em: <https://www.senado.gob. ar/parlamentario/parlamentaria/365901/downloadPdf>. Acesso em: 7 abril 2020.

VELÁZQUEZ-GUTIÉRREZ, José Manuel. Constitucionalismo verde en Ecuador: Derechos de la Madre Tierra y Buen 
Vivir. In: Entramado, v. 10, n. 1, pp. 220-238, 2014. Disponível em: <http:/ / revistas.unilibre.edu.co/index.php/entramado/article/view/3490>. Acesso em: 5 abril 2020.

VIOLA, Francesco. Etica dei diritti. In: VIGNA, Carmelo (org.). Introduzione all'etica. Milano: Vita e Pensiero. pp. 319-338, 2001.

VIOLA, Francesco. Il futuro del diritto. In: Persona y Derecho, n. 79, pp. 9-36, 2018. Disponível em: <https:/ / revistas.unav. edu/index.php/persona-y-derecho/article/view/34238>. Acesso em: 5 abril 2020.

ZAFFARONI, Eugenio Raúl. La naturaleza como persona: de la Pachamama a la Gaia. In: ESPINOSA GALLEGOS-ANDA, Carlos; PÉREZ FERNÁNDEZ, Camilo (orgs.). Los Derechos de la Naturaleza y la Naturaleza de sus Derechos. Quito: Ministerio de Justicia, Derechos Humanos y Cultos. pp. 3-33, 2011a.

ZAFFARONI, Eugenio Raúl. La Pachamama y el humano. In: ACOSTA, Alberto; MARTÍNEZ, Esperanza (orgs.). La naturaleza con derechos. De la filosofía a la política. Quito: Abya-Yala. pp. 25-137, 2011b.

Recebido em 14/04/2020

Aprovado em 18/11/2020

\section{Livio Perra}

E-mail:Iperra@uniss.it 
